\title{
MEASUREMENT BASED LINK LEVEL INVESTIGATION OF IEEE 802.11G CHANNELS
}

\author{
Shaoen $\mathrm{Wu}^{1}$ and Saâd $\mathrm{Biaz}^{2}$ \\ ${ }^{1}$ School of Computing, University of Southern Mississippi, Hattiesburg, MS, USA \\ shaoen. wu@usm. edu \\ ${ }^{2}$ Department of Computer Science and Software Engineering, Auburn University, \\ Auburn, USA \\ biazsaa@auburn.edu
}

\begin{abstract}
Assessing channel conditions is critical to routing and MAC protocols. But, this task is challenging due to the highly dynamic nature of wireless channels and the characteristic differences between indoor and outdoor environments. This paper presents observations and analysis from extensive measurements on IEEE $802.11 \mathrm{~g}$ channels indoor and outdoor. The experiments are designed to investigate the channel dynamics through metrics such as successful frame delivery rate, loss rate, and Signal-to-Noise Ratio on IEEE 802.11 networks. The objective of this work is to reveal the interaction between these metrics and their variations in time and space.

This paper makes the following observations. Frame delivery rate for fixed stations fluctuates over time in most scenarios. Statistics over large time intervals get bursty and do not provide valuable information about channel conditions. Generally, Signal-to-Noise Ratio (SNR) varies widely over time even for fixed stations. However, the SNR remains stable for micro time scales. As a consequence, the frame delivery does not strongly correlate with SNR. Another observation is that indoor and outdoor require different sampling intervals to measure valuable frame loss rate. If sent at a favourable time, a set of frames is likely to be delivered successfully continuously. Although the intermediate distribution of the intervals between consecutive losses varies wildly for different transmission rates (modulations), most of these intervals are less than $5 \mathrm{~ms}$ regardless of the rate and location.
\end{abstract}

\section{KEYWORDS}

Wireless Network, Measurement, IEEE 802.11

\section{INTRODUCTION}

Wireless links are subject to highly variable quality. The channel quality of a wireless link even between static nodes varies over time due to multipath and interferences from other electromagnetic sources. People moving around, opening, and closing doors contribute to such variability. If other nodes are communicating in the vicinity, interference as well as contention exacerbates this variability. Finally, the channel quality may degrade as mobile nodes drift away from each other. This dynamic channel quality results in variable achievable data rates and variable frame loss rates.

Recent developments in wireless network interface cards use techniques based on current channel quality to select the most appropriate data rate. This is called data rate adaptation [1][3]. The most challenging aspect of data rate adaptation is the assessment of the current quality of the channel. The challenge of assessing the channel still remains.

Routing in multi-hop wireless networks is another challenge due to the dynamic nature of wireless channel quality. The selection of an optimal routing path is more difficult than in

DOI : $10.5121 /$ ijcnc.2011.3201 
wired networks even if wireless nodes are static because it is hard to assess the goodness of a path. A variety of routing metrics [4]-[7] aware of link quality/conditions have been proposed for multi-hop wireless networks. The goodness of a path is measured using some metric on each hop. Whatever is the metric on a hop, this metric varies over time with the channel quality. To appreciate the difficulty of the routing problem, consider commuting from home to office in the situation where streets may experience flash flooding and moving riots spark here and there throughout the city.

Therefore, assessing channel quality is paramount to multiple wireless networking problems such as data rate adaptation and routing. The question is how to assess the quality of a channel. Many parameters are considered: Signal to Noise Ratio (SNR), frame loss rate, length of a streak of successful frame transmissions, and number of consecutive frame losses. It is of interest to study how well these parameters characterize or reflect the quality of a channel

This work analyses extensive measurements on a IEEE $802.11 \mathrm{~g}$ channel in indoor and outdoor environments. Our experiments investigate the transient behaviour of the widely used IEEE $802.11 \mathrm{~g}$ channels at $2.4 \mathrm{Ghz}$. The channel dynamics reported in this paper are important to the design of routing metrics, data rate adaptation as well other networking techniques used on IEEE 802.11 networks.

The rest of this paper is organized as following. Section II describes the experimental test bed and methodology. The collected measurements are reported and analysed in Section III. Section IV surveys related work and highlights differences of this work from others.

\section{EXPERIMENTS AND METHODOLOGY}

To capture and analyze indoor and outdoor wireless trans- missions, we designed a measurement platform with a customized packet filter. This section discusses the measurement platform, the experimental environments, the customized soft- ware tool, and the measurement scenarios.

\subsection{Measurement Platform}

As shown in Figure 1, the measurement platform hard- ware consists of three laptops and one access point: Linksys WRT54GS [8]. Each laptop is equipped with an IEEE 802.11 adapter based on the Atheros chipset AR5212. This chipset is functionally supported by the open source driver Madwifi [9] on Linux. The wired client is a system76 darter ultra notebook computer running Ubuntu 8.04 with a dual-core $2.2 \mathrm{GHz}$ Intel processor, 2GB RAM and a Realtek RTL8111 Gigabit Ethernet NIC. The wireless client and the network sniffer are IBM T60 Thinkpad laptops running Fedora Core 9 with dualcore $1.66 \mathrm{GHz}$ Intel processors, 2GB RAM and Atheros AR5212 802.11abg network interface cards. Both Thinkpad laptops use Madwifi ath5k [10] wireless drivers

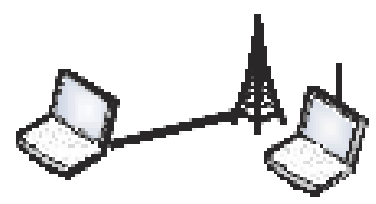

Figure 1: Measurement Platform and all three laptops run Linux kernel 2.6.25.

During the measurements, the access point is static and placed at a height of two meters. One laptop, serving as a sniffer, is placed tightly close to the wireless access point to ensure (as much as possible) that its received wireless signal is similar to the signal received by the sniffer. The sniffer is passive and works in monitor mode in which the 
node does not associate with any access point and does not transmit any frame. With proper modification of the driver, the wireless adapter reports information we are interested in: data rate, channel used, and the received signal strength indication (RSSI). In MadWiFi, the reported RSSI for each frame actually refers to the Signal-to-Noise Ratio (SNR). The network sniffer runs a slightly modified version of tcpdump [11] to monitor the wireless traffic and to gather wireless statistics. For each successfully received frame, the sniffer records and reports its data rate, the channel number, the SNR, the timestamp, the More Frag flag, the Retry flag, the sequence number, the fragment number, and the send/receive flag.

The end-point traffic receiver is a laptop wired to the access point and its wireless adapter is turned off. The third laptop serves as the end-point traffic sender. It sends 1500-byte UDP packets to the wired laptop through the access point. RTS/CTS control frames are turned off. The sending laptop is placed at different locations experiencing distinct signal strengths. All nodes are static during every experiment. The traffic receiver and sender laptops run Iperf [12] for generating or receiving UDP traffic.

For outdoor tests, the wireless channel is set to channel 11 after a preliminary survey of wireless network traffic showed that no other surrounding wireless networks use that channel and hence channel contention would not be an issue. Indoor, we use channel 3 to minimize cochannel interference from surrounding WiFi networks deployed in the building. The transmit power of the endpoint sender is set to its default value of $13 \mathrm{~dB}$ and RTS/CTS control frames are turned off. The fragmentation threshold is set such that fragmentation does not occur during the experiments.

\subsection{Measurement Environments}

We conducted both indoor and outdoor measurements. Indoor measurements were performed on the ground floor of the Shelby building at Auburn University at night during weekends to minimize any interference from walking people. Figure 2 coarsely shows about $1 / 3$ of the floor plan of that building where the experiment was conducted and the layout of the experiment components: the triangle represents the location of the access point and the sniffer placed very close to it. The position of the endpoint laptop receiver is not important because this laptop is connected by wire to the access point and its wireless adapter is off. The circle spots represent the different locations where the sender laptop was placed. Locations are chosen such that the wireless nodes achieve specific maximal data rates. The data rates used are $6 \mathrm{Mpbs}, 24 \mathrm{Mbps}$, and $54 \mathrm{Mbps}$. Locations $L$ 1 and $L-2$ support up to 54 Mbps. Location ( $L-3)$ supports up to $24 \mathrm{Mbps}$. Location $4 L-4$ supports only 6 Mbps.

Locations are similarly selected for outdoor experiments. The outdoor measurements were taken on a wide open space south of campus. The outdoor environment, shown in Figure 3, is free from obstacles like buildings or

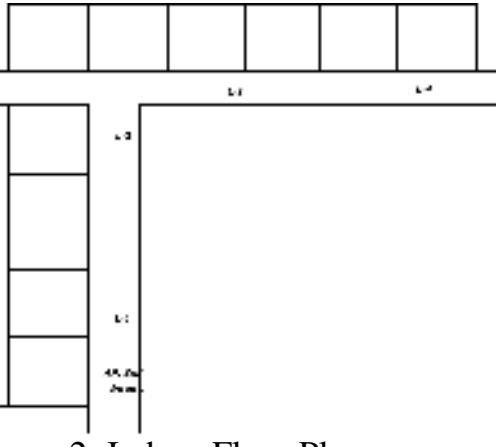

Figure 2: Indoor Floor Plan

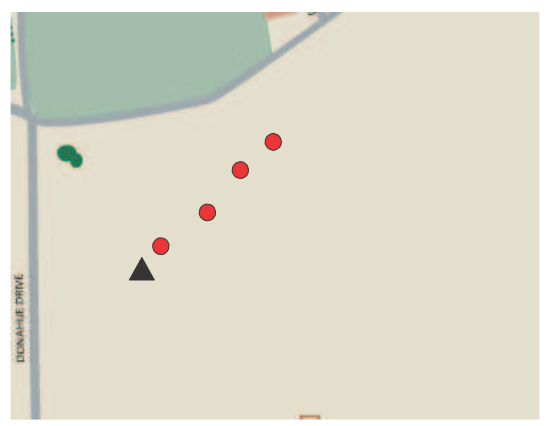

Figure 3: Outdoor Floor Plan trees. The symbols for the outdoor measurement platform layout have the same meaning as the indoor layout in Figure 2. 


\subsection{Customization of Measurement Tools}

Two command line tools are employed in our experiments: tcpdump and Iperf. The network sniffer runs a slightly mod- ified version of tcpdump [11] to monitor the wireless traffic. The endpoint laptops use Iperf [12] to generate or receive UDP traffic.

1) tcpdump: tcpdump [11], developed at the Lawrence Berkeley National Laboratory, is an open source package widely used to capture frames. tcpdump uses the libpcap [13] library for capturing the frames. To customize tcpdump to our needs, we modified tcpdump to output only the information of interest to us. Particularly, we record the data rate, the channel used, the Signal-to-Noise Ratio (SNR), the timestamp, and the following flags: More Frag, Retry, Sequence Control, and Send/Receive. The More Frag flag indicates the latest fragment of a fragmented frame. We use it just to ensure that fragmentation is not occurring. The Retry flag indicates a retransmission. The Send/Receive indicates whether the frame is sent or received. We did not change the capturing functionality of tcpdump, but only the output format and content. tcpdump was configured to capture only data frames and ignore control/management for two reasons: 1) data frames are strictly sequential so losses are easy to identify, and 2) less storage space is required for the trace. Figure 4 highlights the difference between the original tcpdump output and the modified version's. Moreover, a MAC address filter is exploited to collect only frames transmitted from the MAC address of the wireless sender so that other traffic from the router such as beacons, association requests, and other management frames are ignored. All log files are recorded in a customized plain text format.

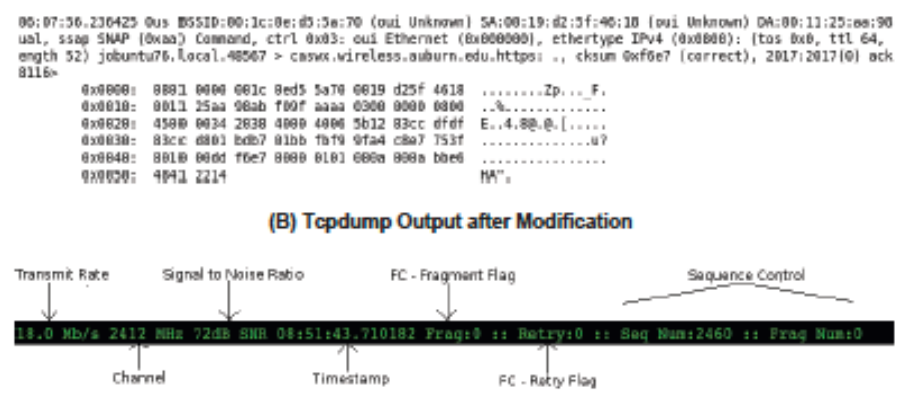

Figure 4: Customized TCPDUMP output

2) Iperf: Iperf [12] is used as the UDP traffic generator and sink for the measurements. The wired endpoint receiver runs the Iperf sink to receive the generated UDP traffic. Iperf on the wireless endpoint sender generates the UDP traffic towards the sink. Iperf is set such that during each experiment the traffic flow lasts 60 seconds and that the sending rate is always $1 / 3$ of the maximal achievable wireless data rate. For example, if the highest achievable rate is $24 \mathrm{Mbps}$ on the wireless link, Iperf generates $8 \mathrm{Mbps}$ traffic so that losses do not occur at the sender's output queue.

\subsection{Measurement scenarios}

Experiments indoor and outdoor use the combinations of four distances and three data rates. Please, note that some locations do not support high data rates. The detailed combinations of data rates and locations are illustrated in Table I. In total, measurements were made for seven test scenarios. Each test scenario is repeated six times in order to ensure that enough measurements are collected.

\begin{tabular}{|l|c|c|c|c|}
\hline Location & 1 & 2 & 3 & 4 \\
\hline Data Rates (Mbps) & $6,24,54$ & $6,24,54$ & 6,24 & 6 \\
\hline
\end{tabular}

Table I: Data Rate Combination 


\section{Measurements ANd Analysis}

This section presents our observations and analysis based on the traces collected through the extensive indoor and outdoor measurements.

\subsection{Variations of Delivery Ratio}

First, we analyze the variations of the frame delivery ratio over time. The frame delivery ratio is the ratio of the number of frames successfully received over the number of frames that should have been during an interval of $100 \mathrm{~ms}$. The number of frames that should have been received is determined from the sequence control field on the IEEE 802.11 frame header. The delivery ratios for outdoor and indoor are respectively shown on Figure 5 and 6 . The y-axis represents the delivery ratio averaged over the six runs for each data rate and the x-axis is time. We trim the very beginning and the end of the trace to eliminate large variations due to startup and completion of an experiment. When the wireless signal is stable and reliable at Locations 1 and 2 in both environments, the delivery ratio at each data rate remains relatively steady. But it fluctuates wildly for $24 \mathrm{Mbps}$ outdoor. In accordance with wireless communication theory, the delivery ratio decreases as the data rate increases at a particular location. Note that the overall throughput (the product of data rate and delivery rate) at rate $54 \mathrm{Mbps}$ is the best even though its delivery ratio is the worst. Delivery ratios get more irregular as distance increases as shown on the plot for Location 3 indoor and outdoor. This implies that the delivery ratio alone is not a robust indicator of channel conditions.
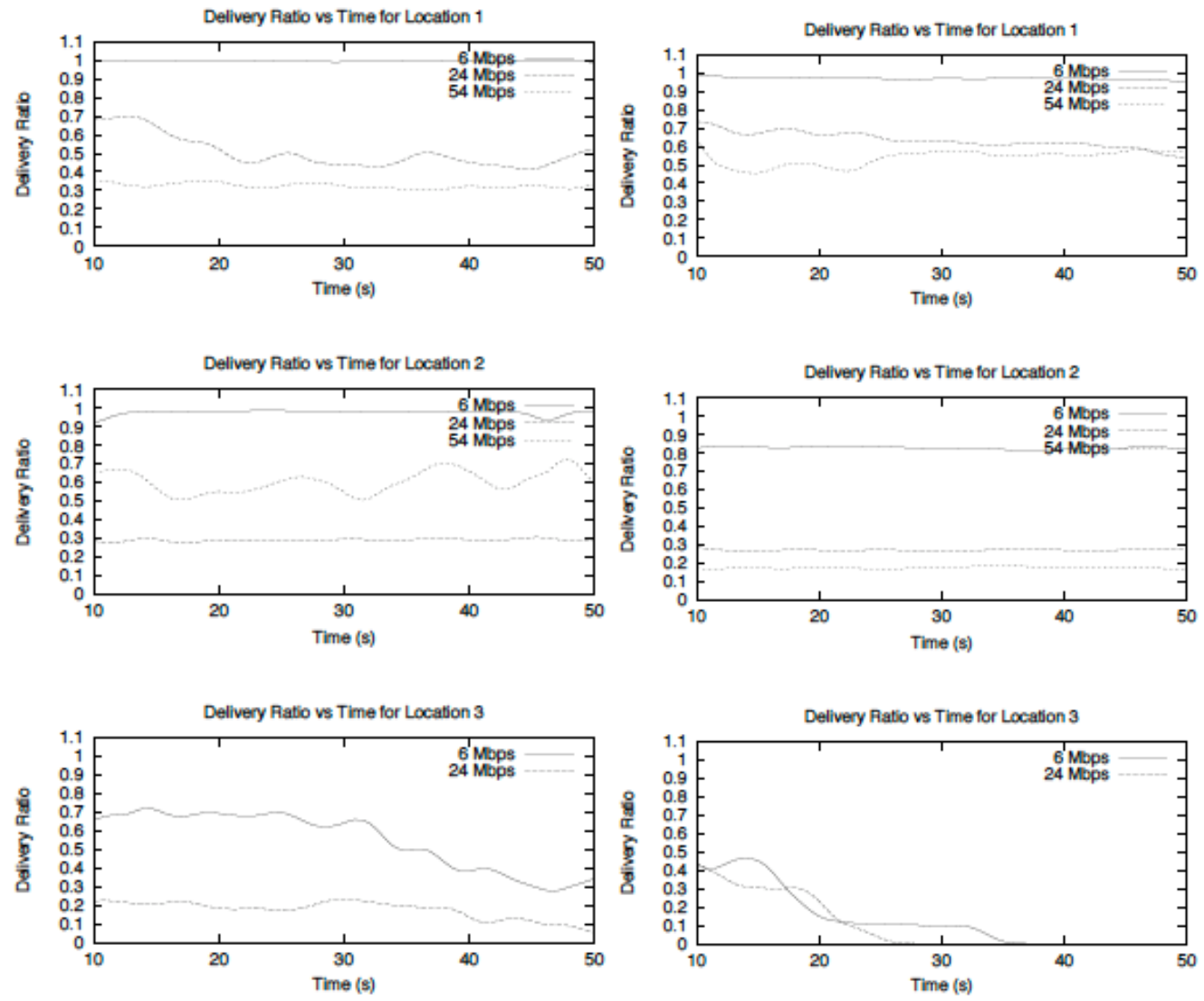

Figure 5: Delivery Ratios vs Time Outdoor

Figure 6: Delivery Ratios vs Time Indoor 


\subsection{Allan Deviation of Loss Rate}

Section III-A illustrates the variation of delivery ratio measured over intervals of $100 \mathrm{~ms}$. The delivery ratio is quite correlated with the loss rate that we also measured. It is of interest to study whether the sampling interval would impact the loss rate measurements and ultimately our conclusions. One approach to such analysis is the plot of the Allan deviation [14] for frame loss rate. The Allan deviation could reveal the bursty nature of loss rate. Whereas standard deviation captures the difference between a sample and the mean, the Allan deviation uses the difference between two consecutive samples. In our case, the samples are the loss rates in consecutive intervals. We studied the Allan deviation using different interval values on the same trace. The Allan deviation of a sequence of samples of li is calculated as:

$$
A D=\sqrt{\frac{1}{2 n} \sum_{i=2}^{n}\left(x_{i}-x_{i-1}\right)^{2}}
$$

When the time interval is close to the characteristic burst size of the loss rate, its Allan deviation is large. For very small or large intervals, the Allan deviation is small. Therefore, the Allan deviation is useful in identifying the time interval for which the loss rate varies most from sample to sample. The information is valuable for statistics gathered over intervals yielding a small Allan deviation.

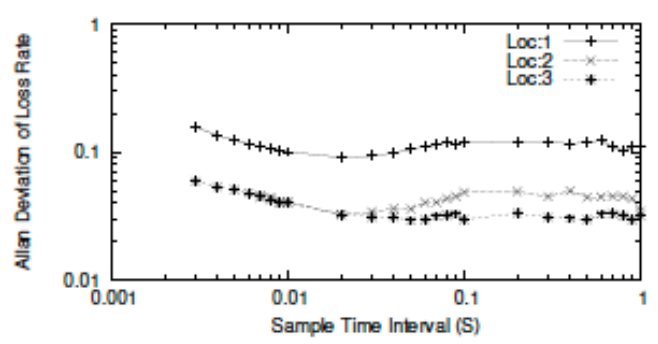

Figure 7: Allan Deviation Outdoor

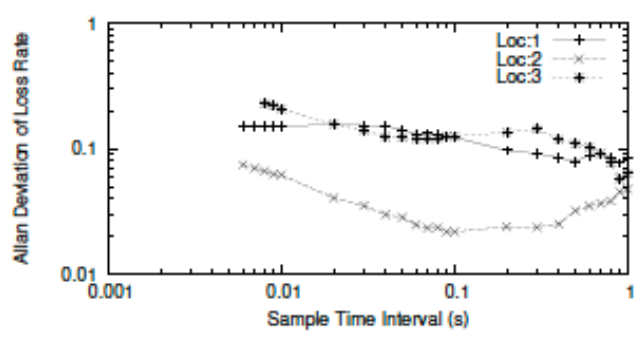

Figure 8: Allan Deviation Indoor

Figure 7 and Figure 8 respectively show the Allan deviation of the loss rates in outdoor and indoor environments from our measurements. Each line corresponds to one location. During our experiments for Location 4, only a few frames are successfully received. Therefore, the Allan deviation for Location 4 is omitted. The Allan deviation that is plotted comes from measurements made at a data rate of $24 \mathrm{Mbps}$. The $\mathrm{x}$-axis shows the intervals duration over which the loss rate is averaged. The y-axis stands for the Allan deviation. From these figures, the Allan deviations decrease when the time intervals are smaller than 100 milliseconds for indoor and 20 milliseconds for outdoors. The increase of the Allan deviations at large time intervals implies that the loss rate is burstier and less informative.

\subsection{Variations of Signal-to-Noise Ratio}

The SNR variations over time are plotted in Figure 9 and Figure 10, respectively for outdoor and indoor. The data shown in these figures are from the trace at $24 \mathrm{Mbps}$. The y-axis represents the SNR measured in each frame. The general observation is that in both outdoor and indoor environments the SNR varies irregularly for different distances: especially for the farthest location inside the building, the SNR varies wildly over time. The large fluctuations may be due to multi-path fading. As a result, SNR alone is not reliable to assess channel quality or conditions. 
To obtain more details about SNR variations over time, we plot a small fraction of a 60 -second trace outdoor and indoor in Figures 11 and 12, respectively. Since only a few frames were collected at Location 4 indoor, we omit the monitored SNR. Location 3 indoor can only support frames at $6 \mathrm{Mbps}$. Therefore, for consistency in the figures, we plot 3 locations for indoor and outdoor where the farthest location was tested at $6 \mathrm{Mbps}$. The SNR seems stable among consecutive frames except for Location 1 indoor where it varies in saw shape. Since Location 1 is the closest to the measurement station, it has a large SNR mean. Even though the SNR has a large absolute standard deviation, the relative standard deviation (RSD) (i.e, the ratio of the standard deviation over the mean) is still small. Numerically, for the outdoor case, the three locations respectively have SNR means of $56.95 \mathrm{~dB}, 36.74 \mathrm{~dB}$, and $26.69 \mathrm{~dB}$, and standard deviations of $4.16 \mathrm{~dB}, 1.53 \mathrm{~dB}$ and $0.766 \mathrm{~dB}$. Indoor, the SNR means are $47.85 \mathrm{~dB}, 42.11 \mathrm{~dB}$ and $24.23 \mathrm{~dB}$, and the standard deviations are $0.69 \mathrm{~dB}, 1.75 \mathrm{~dB}$ and $1.59 \mathrm{~dB}$ for each location.

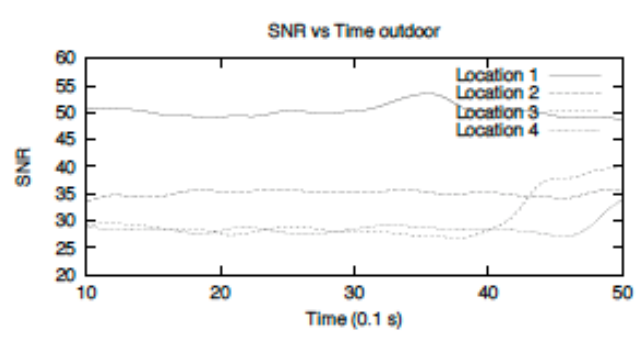

Figure 9: Variation of SNR Outdoor

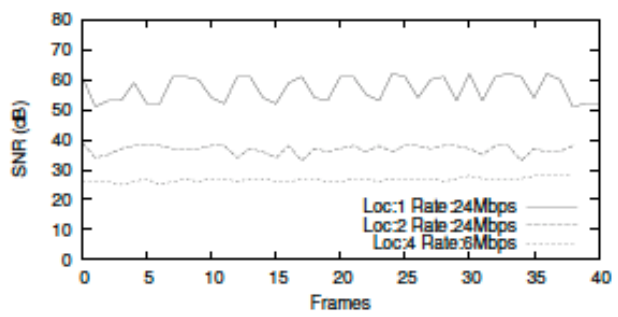

Figure 11: Micro Variation of SNR Outdoor

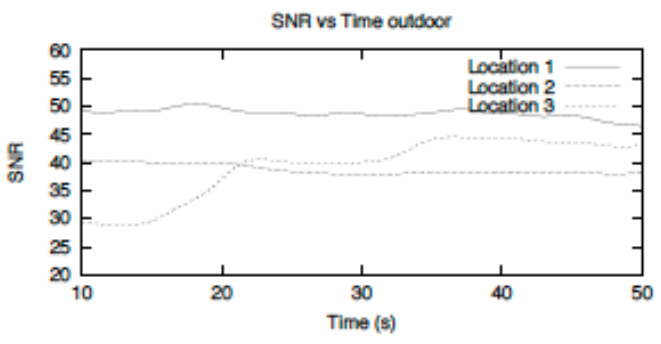

Figure 10: Variation of SNR Indoor

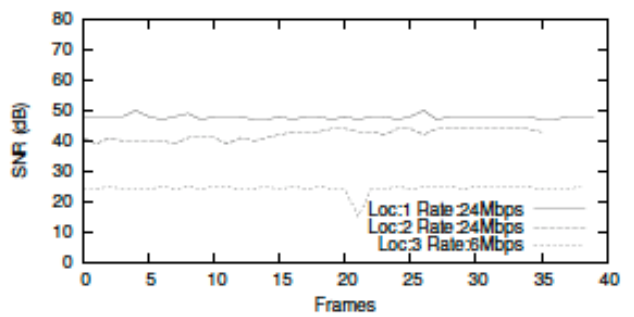

Figure 12: Micro Variation of SNR Indoor

\subsection{Delivery Rate and Signal-to-Noise Ratio}

Using the same trace as in Section III-A, we plot the relationship between delivery ratio and signalto-noise ratio (SNR) to find any correlation between them. The statistics are computed at intervals of $100 \mathrm{~ms}$. Figure 13 and Figure 14 respectively depict outdoor and indoor measurements at $24 \mathrm{Mbps}$ at Location 1. The yaxis refers to the delivery ratio. The $\mathrm{x}$-axis represents the SNR averaged over a $100 \mathrm{~ms}$ period. Each symbol in the figures represents a transmission sample. One common observation from these plots is that there is no strong correlation between the delivery ratio and SNR: we often observe very different delivery ratios at the same SNR. Similarly, we observe the same delivery rate at very different SNR values. In conclusion, SNR alone is not a robust indicator for

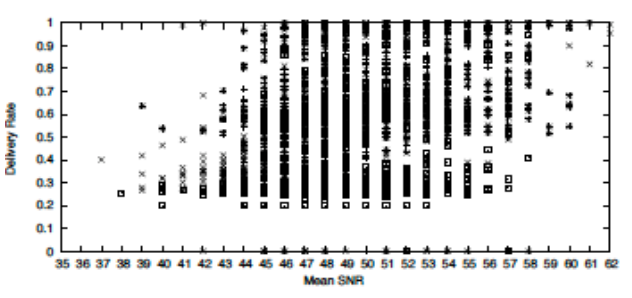

Fig. 13: Delivery Ratios vs SNR Outdoor

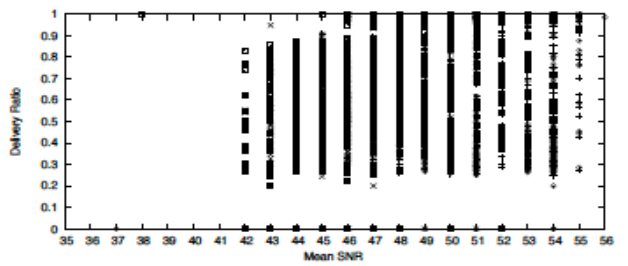

Figure 14: Delivery Ratios vs SNR Indoor 
channel condition. From these figures, the SNR indoor is more concentrated and with shorter tail than outdoor.

\subsection{Consecutive transmissions}

We analyze the cumulative distribution function (CDF) of the number of consecutive successful transmissions to investigate the sustainability of transmissions at a given data rate. Figure 15 and Figure 16 respectively depict indoor and outdoor measurements. The y-axis shows the CDF and the $\mathrm{x}$-axis stands for the number of consecutive successful transmissions between two transmission failures. Each line in these figures represents one collection of transmissions at $6 \mathrm{Mbps}$ at Location 1 or 2 . The inner magnified figures show that only a small fraction of streaks with a low number of consecutive transmissions (the left most on the x-axis). Namely, we observe that outdoor (resp. indoor), less than $40 \%$ (resp. 15\%) of streaks have less than 4 consecutive successful transmissions. In other words, most of time the channel can support transmission of more than 4 frames consecutively. It should be noted that these results are obtained in stable environments without interference. Mobility should have some impact on this observation. The difference in CDF between indoor and outdoor is critical for the design of an effective unified rate adaptation strategy.

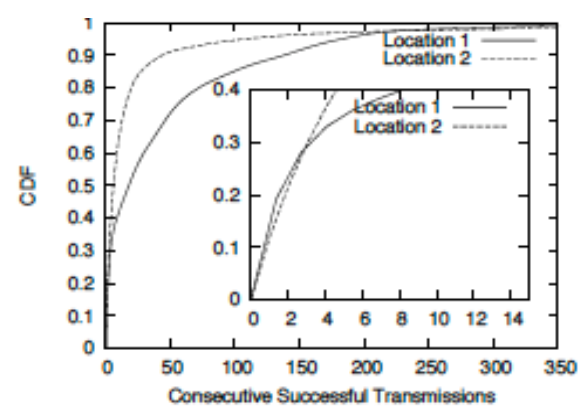

Figure 15: CDF Outdoor

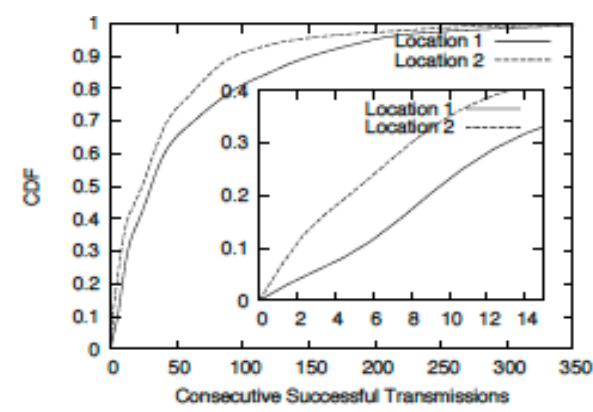

Figure 16: CDF Indoor

\subsection{Time Variation of Frame Loss}

After investigating the number of consecutive successful transmissions in the above section, we focus in the next two sections on the transient behavior of loss rates. First, the variation of frame loss over time at different locations is inspected.

In practical wireless communication, wireless channels can be modeled as time variant systems. Therefore, it should be interesting to understand the frame loss pattern. For this purpose, we inspect the distribution of the time interval between two consecutive frame losses. Figure 17 and Figure 18 respectively plot the cumulative distribution function (CDF) of the time between two consecutive losses for outdoor and indoor environments. The trace contains the traffic at 24 Mbps collected from different locations. Outdoor, most of the losses occur less than 3 milliseconds after a loss: over 95\% (Locations 1 and 2) and about 80\% (Location 3). Because of multi-path fading, the indoor environment is slightly different. About $80 \%$ of intervals fall into durations less than $1 \mathrm{~ms}$ for Locations 1 and 2, but less than 20 millisecond for Location 3.

\subsection{Impact of Transmission Rates on Frame Loss}

It is of interest to investigate the impact of the data rate on the CDF of the time between two consecutive losses at various locations. This section presents in Figure 19 and Figure 20 the $\mathrm{CDF}$ computed at different rates outdoor and indoor. Data are from measurements carried out at Location 2 indoor and outdoor. Each curve represents the result at different data rates. The yaxis in these figures shows the $\mathrm{CDF}$ and the $\mathrm{x}$-axis represents the time interval between two 
International Journal of Computer Networks \& Communications (IJCNC) Vol.3, No.2, March 2011

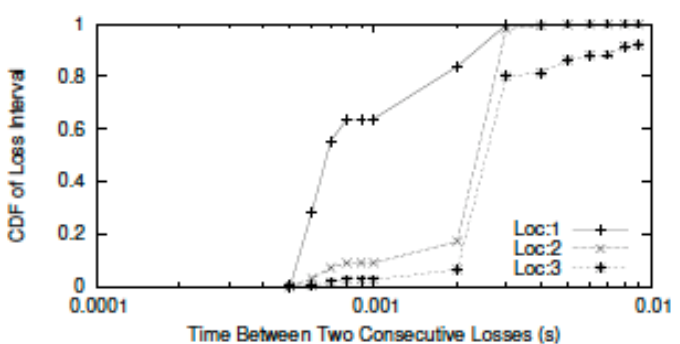

Figure 17: Variation of Loss Rate Outdoor

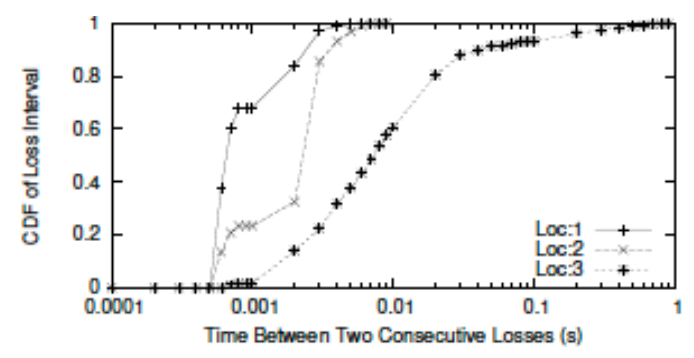

Figure 18: Variation of Loss Rate Indoor

consecutive losses. From these figures, most losses occur less than $2 \mathrm{~ms}$ apart: over $95 \%$ for outdoor and about $80 \%$ for indoor. It seems that the statistics of loss rate within 5 ms should contain most information reflecting the variations of the wireless channels for IEEE 802.11 networks indoor and outdoor. Also, the distribution of the loss interval varies for different rates. Most of the losses regularly occur within 1 to $2 \mathrm{~ms}$ indoor and outdoor. But, at higher rates the loss intervals span large time intervals. This might be due to more robust modulation schemes at lower rates that are more resilient to the time variations of the channel conditions up to a certain critical frequency at which most of frame losses occur.

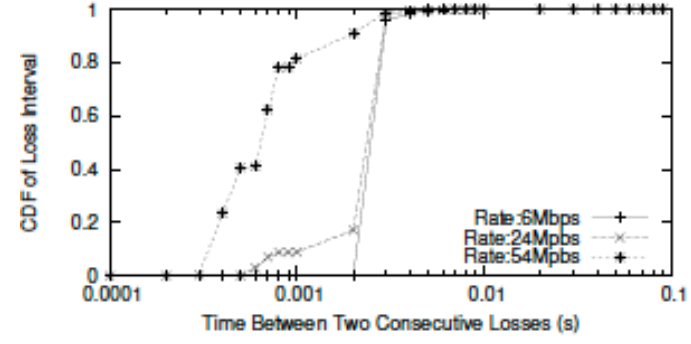

Figure 19: Impact of Rate Outdoor

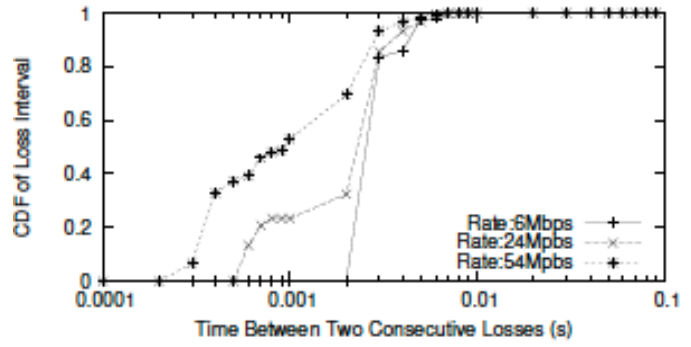

Figure 20: Impact of Rate Indoor

\section{RELATED WORK}

This section presents some measurements performed on IEEE 802.11 networks in literature.

Rodrig et al. [15] carried measurements of 24 hours during the SIGCOMM 2004 conference on IEEE $802.11 \mathrm{~b}$ channels. They analyzed the transmission efficiency of IEEE 802.11MAC. Their results show that the rate adaptation schemes used at the time were inefficient: only $40 \%$ of the transmission time is spent in sending new data frames. Most of the remaining

time is used by retransmissions due to frame losses by contention or channel degradation. They also observe that the rate adaptation schemes in commercial IEEE 802.11 adapters switch their rates too frequently. Bianchi et al. [16] compared the channel performance between IEEE $802.11 \mathrm{~b}$ and IEEE 802.11g. They conducted their measurements on an outdoor campus network. They observed that these two standards behave noticeably differently even under the same environment. They attributed this difference to the physical layer coding schemes. Aguayo et al.[17] analyze the causes of frame loss in an outdoor IEEE $802.11 \mathrm{~b}$ mesh network called roofnet [18] on MIT Cambridge campus. This network contains multiple mesh routers statically placed on the roof of buildings. They observe that most nodes experienced intermediate loss rates in spite of the difference among the distances between various node pairs, somehow contradicting the intuitive expectation.

Therefore, there is no clear cut-off loss rate threshold for nodes that are "in range" or "out of range". One of their observations is that signal-to-noise ratio (SNR) and distance are rarely indicative of the loss rate. They infer that the main contributor to intermediate loss rates is 
multi-path fading. One similar work is conducted by Gupta [19]. However, in long-range measurements, outdoor IEEE 802.11b WLAN, Chebrolu et al. [20] found that the loss rate changes as a function of received signal strength. Part of the reason of the discrepancy with Aguayo et al. comes from the fact that their network have long distance links and a sparse base station distribution. Each link operates on non-overlapping IEEE 802.11 channels. As a result, contention losses are rare. This network layout is fundamentally different from Aguayo's [17] where collisions are likely to occur because of the dense mesh routers. Similar observations are obtained by Gokhale, et al. [21]. Another long-distance link measurement work is conducted by El-Sayed, Zeadally and Boulmalf [22]. This work summarizes the characterization of this kind of links. They also studied the relationship between SNR and throughput of IEEE 802.11g link in a small enterprise environment [23]. Kotz et al. [24] set out to verify hypothesis about radio propagation that many network simulation models are based upon. The commonly accepted assumptions include: the wireless signal is propagated in the circular shape; all signals have equal transmission range; symmetric links; and signal strength is a simple function of communication distance. They carried out a set of IEEE $802.11 \mathrm{~b}$ measurements in both indoor and outdoor environments. They observe that these assumptions do not adequately match real world behavior, which severely undermines the accuracy of the conclusions drawn from simulations based on such assumptions. Cheng et al. [25] conducted measurements to study the impact of the antenna orientation to an unmanned aerial vehicle (UAV). They equipped the UAV and the ground stations with adapters operating on IEEE 802.11a channel. They conclude that to achieve the best throughput performance, both antennas of the UAV and the ground station should be omnidirectional and placed horizontally with their null pointing to a direction perpendicular to the flying path. Finally, Giustiniano, et al. [26] observed that the hardware/software diversity at a transmitter may induce weird behavior of wireless signal at a receiver.

Our work is different from the above measurements in several points. First, this work is dedicated to the investigation of channel dynamics fundamentally impacting on IEEE 802.11 communications. The "mystery" revealed could benefit various fields, such as routing, MAC protocols, and rate adaptation. Second, the measurements are based on the IEEE 802.11g that is widely being used in commercial products on the market, although most of the above measurements conducted on IEEE 802.11b that is obsolete. Third, most of above measurements are conducted in either indoor or outdoor. Our work investigates indoor and outdoor using the same test bed. The limitation of this work is the lack of measurements in presence of collisions. This will be on our agenda for future work.

\section{Conclusions}

This work presents and discusses observations about IEEE 802.11 channel dynamics obtained from extensive outdoor and indoor measurements conducted on a customized test bed. Particularly, we investigate space and time variations as well as relationships of frame delivery rate, loss rate, and Signal-to-Noise Ratio. We observe that neither frame delivery rate nor SNR alone accurately reflects channel variations because they fluctuate over time. Frame delivery rate and SNR are not strongly correlated. Large time scale measurements do not yield informative or reliable statistics. We also observe that indoor environments require different sampling intervals from outdoor to measure a meaningful frame loss rate. Note that the CDF of the time interval between losses varies wildly based on the data rate and distance between nodes. However, a large proportion of the intervals between losses are less than $5 \mathrm{~ms}$ regardless of the data rate and distance. These observations are valuable for designing routing and MAC strategies/protocols for wireless networks. 
International Journal of Computer Networks \& Communications (IJCNC) Vol.3, No.2, March 2011

\section{ACKNOWLEDGEMENTS}

This work is funded by the National Science Foundation through grants OCI\#1041292, NeTS CNS\#0435320, CRCD/EI CNS\#0417565 and REU CNS\#0552627.

\section{REFERENCES}

[1] J. Kim, S. Kim, S. Choi and D. Qiao, "CARA: Collision-Aware Rate Adaptation for IEEE 802.11 WLANs,” in IEEE INFOCOM’06, Barcelona, Spain, April 2006.

[2] S. Wong, H. Yang, S. Lu and V. Bharghavan, "Robust Rate Adaptation for 802.11 Wireless Networks," in MobiCom'06, Angeles, California, USA., September 2006, pp. 146 - 157.

[3] S. Biaz and S. Wu, "ERA: Effective Rate Adaptation for WLANs," in IFIP Networking 2008. Singapore: IFIP, May 2008.

[4] D. S. J. De Couto, D. Aguayo, J. Bicket, and R. Morris, “A high-throughput path metric for multi-hop wireless routing," Wirel. Netw. vol. 11, no. 4, pp. 419-434, 2005.

[5] Biswas, Sanjit and Morris, Robert, "ExOR:Opportunistic Multi-Hop Routing For Wireless Networks," in SIGCOMM Comput. Commun. Rev., vol. 35, no. 4, New York, NY USA, 2005, pp. 133-144.

[6] R. Draves, J. Padhye, and B. Zill, "Comparison of routing metrics for static multi-hop wireless networks," in SIGCOMM '04: Proceedings of the 2004 conference on Applications, technologies, architectures, and protocols for computer communications. New York, NY, USA: ACM, 2004, pp. 133-144.

[7] R. Draves, J. Padhye, and B. Zill, "Routing in multi-radio, multi-hop wireless mesh networks," in MobiCom '04: Proceedings of the 10th annual international conference on Mobile computing and networking. New York, NY, USA: ACM, 2004, pp. 114-128.

[8] Linksys. [Online]. Available: http://www.linksysbycisco.com/US/en/support/WRT54GS

[9] Madwifi. [Online]. Available: http://madwifi.org/

[10] madwifi. [Online]. Available: http://linuxwireless.org/en/users/Drivers/ath5k

[11] TCPDUMP. [Online]. Available: http://www.tcpdump.org/

[12] Ajay Tirumala, Feng Qin, Jon Dugan, Jim Ferguson, and Kevin Gibbs. [Online]. Available: http://dast.nlanr.net/Projects/Iperf/

[13] [Online]. Available: http://www.tcpdump.org/

[14] D. W. Allan, "Time and frequency (time-domain) characterization, estimation, and prediction of precision clocks and oscillators," vol. 34, no. 6, pp. 647-654, 1987.

[15] Maya Rodrig, C. Reis, R. Mahajan, D. Wetherall, and J. Zahorjan, "Measurement-Based Characterization of 802.11 in a Hotspot Setting," in ACM SIGCOMM Workshops, 2005.

[16] Giuseppe Bianchi, Fabrizio Formisano, and Domenico Giustiniano, "802.11b/g Link Level Measurements for an Outdoor Wireless Campus Network,” in WoWMoM’06, 2006.

[17] D. Aguayo, J. Bicket, S. Biswas, G. Judd, and R. Morris, "Link-Level Measurements from an 802.11b Mesh Networks," in ACM SIGCOMM, 2004.

[18] Dan Aguayo, John Bicket, Sanjit Biswas and Robert Morris, "Mit roofnet outdoor networks." [Online]. Available: http://pdos.csail.mit.edu/roofnet/doku.php

[19] P. Gupta, B. Jain, B. Raman, and P. Kulkarni, "Link-level measurements of outdoor 802.11g links," in Proc. 6th Annual IEEE Communications Society Conf. Sensor, Mesh and Ad Hoc Communications and Networks Workshops SECON Workshops '09, 2009, pp. 1-6.

[20] Kameswari Chebrolu, Bhaskaran Raman, and Sayandeep Sen, "Long-Distance 802.11b Links: Performance Measurements and Experience,” in Mobicom'06, 2006. 
International Journal of Computer Networks \& Communications (IJCNC) Vol.3, No.2, March 2011

[21] D. Gokhale, S. Sen, K. Chebrolu, and B. Raman, "On the feasibility of the link abstraction in (rural) mesh networks," in Proc. INFOCOM 2008. The 27th Conf. Computer Communications. IEEE, 2008, pp. 61-65.

[22] H. El-Sayed, S. Zeadally, and M. Boulmalf, "Experimental evaluation and characterization of long-distance 802.11g links," in Proc. Seventh Int. Conf. Networking ICN 2008, 2008, pp. 511516.

[23] M. Boulmalf, H. El-Sayed, and A. Soufyane, "Measured throughput and snr of ieee 802.11g in a small enterprise environment," pp. 1333-1337, 2005, vehicular Technology Conference, 2005. VTC 2005-Spring. 2005 IEEE 61st.

[24] D. Kotz, C. Newport, R. S. Gray, J. Liu, Y. Yuan, and C. Elliott, "Experimental evaluation of wireless simulation assumptions," in MSWiM '04: Proceedings of the 7th ACM international symposium on Modeling, analysis and simulation of wireless and mobile systems. New York, NY, USA: ACM, 2004, pp. 78-82.

[25] Chen-Mou Cheng, Pai-Hsiang Hsiao, H. T. Kung, and Dario Vlah, "Performance Measurement of 802.11aWireless Links from UAV to Ground Nodes with Various Antenna Orientations," in ICCCN'06, 2006.

[26] D. Giustiniano, G. Bianchi, L. Scalia, and I. Tinnirello, "An explanation for unexpected 802.11 outdoor link-level measurement results," in Proc. INFOCOM 2008. The 27th Conf. Computer Communications. IEEE, 2008, pp. 2432-2440.

\section{Authors}

Shaoen Wu received a Ph.D. in Computer Science in 2008 from Auburn University. He is presently an Assistant Professor of School of Computing at the University of Southern Mississippi. He has held researcher scientist position at ADTRAN Inc. and senior software engineer position at Bell Laboratories. His current research is in the areas of wireless networking, mobile computing and cyber security. He is a recipient of Best Paper Award of ISCC 2008. He has served as chairs and on the committees of several conferences and as editor for several journals. More information is at http://www.cs.usm.edu/ swu.

Saâd Biaz (M '98) received a Ph.D. in Computer Science in 1999 from Texas A\&M University and a Ph.D. in Electrical Engineering in 1989 from the University Henri Poincarè in Nancy (France). He is presently an Associate Professor of Computer Science and Software Engineering at Auburn University. He has held faculty positions at the Ecole Supèrieure de echnologie de Fès and Al Akhawayn University in Ifrane (Morocco). His current research is in the areas of distributed systems, wireless networking, and mobile computing. His research is funded by the National Science Foundation. He is a recipient in 1995 of the Excellence Fulbright Scholarship. He has served on the committees of several conferences and as editor for

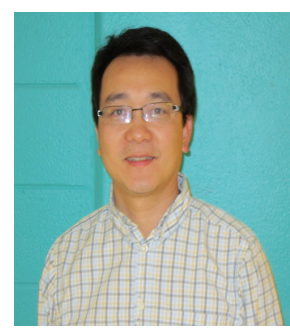
several journals. For more information, please visit http://www.eng.auburn.edu/users/sbiaz.

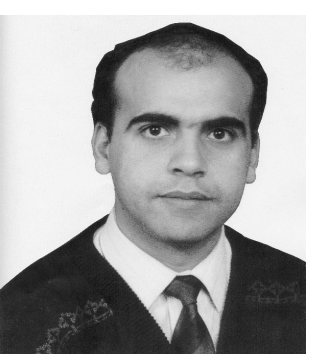

